\title{
Clinical Reasoning: A 23-year-old woman with fever and vertical diplopia
}

David J. Lin, MD, Seth N. Levin, MD, Catherine S.W. Albin, MD, Anna E. Goodheart, MD, and Nagagopal Venna, MD

Neurology ${ }^{\circledR}$ 2018;90:e2006-e2010. doi:10.1212/WNL.0000000000005600

\section{Section 1}

A 23-year-old woman with a history of uncomplicated migraine headaches presented with 2 days of headache. Her typical migraines were preceded by visual aura and usually resolved after taking a triptan. This headache was different in that there was no preceding aura, it did not respond to Imitrex, and it was accompanied by nausea/vomiting, dizziness, subjective fevers, and binocular vertical diplopia. She had had a mild upper respiratory tract infection 1 week prior to presentation. She had no sick contacts or recent travel history. Initial bedside examination showed hypertropia OD (figure 1) that did not change with direction of gaze. The degree of hypertropia improved with supine positioning. Her extraocular movements were otherwise not restricted, and her pupillary responses were normal. She had no other abnormalities on her neurologic examination.

\section{Questions for consideration:}

1. How would you describe the ocular abnormality?

2. What is the expected localization?

3. What is the differential diagnosis?

\author{
Correspondence \\ Dr. Lin \\ Dlin7@partners.org
}

Figure 1 Skew deviation

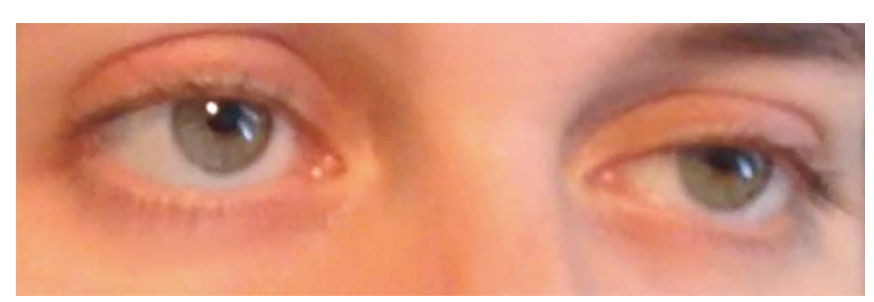

A 23-year-old woman presented with vertical diplopia and was found to have a hypertropia OD consistent with skew deviation. 


\section{Section 2}

Our patient's vertical diplopia, which occurred in all positions of gaze and improved with supine positioning, was most consistent with skew deviation. Skew deviation is a vertical misalignment of the eyes that results from a supranuclear disruption of conjugate vertical eye movements. It results from damage to the vestibulo-ocular system, which normally maintains eye fixation during head movements.

The otoliths (saccules and utricles) and semicircular canals detect linear and angular acceleration, respectively, coordinating vertical eye movement in response to head position. Projections from the utricle and semicircular canals synapse on vestibular nuclei. Second order fibers cross the midline, ascend via the medial longitudinal fasciculus (MLF), and innervate the third and fourth cranial nerve nuclei, the rostral interstitial nucleus of the MLF, and interstitial nucleus of Cajal. The latter nuclei reside in the midbrain and coordinate vertical gaze. An injury to any part of this pathway, spanning the peripheral nervous system and CNS, can cause skew deviation. Lesions in different locations classically result in types of skew as delineated by careful clinical observation. For example, lesions of the utricle result in upward deviation of both eyes with different amplitudes; lesions in the dorsolateral medulla result in hypertropia of one eye with the other eye remaining in the primary position; and lesions in the midbrain tegmentum result in simultaneous hypertropia of one eye and hypotropia of the other eye. ${ }^{1}$

The combination of head tilt, ocular torsion, and skew deviation is known as the ocular tilt reaction. Head tilt is a maneuver to compensate for a shifted vertical meridian. For example, a lesion in the left utricle causes the brain to perceive that the head is tilting to the right. The head rotates in the counterclockwise direction (leftward) to compensate. Lesions in the utricle, caudal brainstem, or cerebellum usually result in ipsilateral head tilts, while lesions rostral to the midpons result in contralateral head tilt.

Ocular torsion or cyclotorsion describes rotation about the visual axis of the eyes and results from aberrant nuclear control of extraocular muscles. Third nerve subnuclei normally control ipsilateral inferior rectus and inferior oblique muscles. The third nerve superior rectus subnucleus and trochlear nucleus control the contralateral superior rectus and superior oblique muscles, respectively. As a result, injury along the MLF will cause the ipsilateral eye to appear hypertropic (inferior rectus palsy) and incyclotorted (inferior oblique palsy), and the contralateral eye to appear hypotropic (superior rectus palsy) and excyclotorted (superior oblique palsy). Notably, if the lesion causing skew occurs before the vestibular-ocular projections cross the midline and join the MLF (i.e., in the vestibular apparatus or pontomedullary junction), the ipsilateral eye will be hypotropic and excylcotorted, and the contralateral eye will be hypertropic and incyclotorted. ${ }^{2}$

The hypertropia of skew deviation is generally the same in all positions of gaze, i.e., comitant, which usually differentiates it from a fourth nerve palsy. However, this is not always the case as there can be incomitant skew deviations. Another distinguishing maneuver between skew deviation and trochlear nerve palsy is the upright-supine test. The vertical misalignment of a skew deviation should decrease by at least $50 \%$ when the patient moves from an upright to supine position. ${ }^{3}$ This likely results from a shift in the utricular axis during the transition from supine to upright. Since utriculo-vestibular pathways are not affected by infranuclear lesions, the same change in vertical misalignment with change in position should not be seen with a fourth nerve palsy.

Our patient's skew deviation accompanied by nausea and vomiting best localized to the caudal part of the floor of the fourth ventricle along the area postrema. She did not have a clear head tilt and cyclotorsion was difficult to detect on bedside testing, but the comitant hypertropia with positive upright-supine testing pointed away from a fourth nerve palsy, and allowed us to diagnose skew and localize supranuclearly.

The differential for the patient's clinical presentation was broad and included vascular, neoplastic, inflammatory, and infectious processes. A brainstem stroke could cause skew deviation, but would be unlikely in this previously healthy young woman with no vascular risk factors. Posterior fossa tumors, in particular ependymoma, can present with isolated brainstem signs. In a young woman, demyelinating conditions such as multiple sclerosis and neuromyelitis optica require consideration. Given her history of subjective fevers and the recent upper respiratory tract infection, inflammatory and infectious etiologies with a predilection for the brainstem such as listeria, mycoplasma, tuberculosis, fungal infections, and atypical viral infections (i.e., varicella-zoster virus [VZV], herpes simplex virus [HSV], and West Nile virus) were at the top of our differential diagnosis.

\section{Question for consideration:}

1. What further diagnostic tests would be helpful?

GO TO SECTION 3 
Table CSF profile at 2 different time points during hospitalization

\begin{tabular}{lll}
\hline & Hospital day 1 & Hospital day 4 \\
\hline Cell count & 20 (70\% neutrophils) & $200(75 \%$ lymphocytes $)$ \\
\hline Glucose & 73 & 55 \\
\hline Protein & 44 & 67 \\
\hline
\end{tabular}

\section{Section 3}

Initial brain MRI showed no specific abnormalities to explain the patient's symptoms or examination findings. On hospital day 1 , she spiked fevers to $103^{\circ} \mathrm{F}$. Her CSF was notable for a neutrophilic pleocytosis with normal protein and glucose (table).

On hospital day 3, the patient developed new flaccid right arm weakness. A repeat brain MRI revealed diffuse T2 hyperintensities, which involved the floor of the 4 th ventricle. Spine
MRI showed longitudinally extensive T2 hyperintensities along the anterior horn of the cervical cord (figure 2). This anterior cord lesion likely explained her arm weakness.

A repeat lumbar puncture on hospital day 4 showed an increased pleocytosis with a shift from a neutrophilic to lymphocytic predominance and elevated protein (table).

A broad infectious workup was notable for a positive serum Mycoplasma pneumoniae immunoglobulin M ( $\operatorname{IgM}$ ) with a positive confirmatory immunofluorescence. CSF PCR for mycoplasma was negative. Negative infectious workup included negative bacterial and fungal cultures as well as negative dedicated testing for HSV, VZV, Epstein-Barr virus, HIV, echovirus, syphilis, Lyme, Cryptococcus, West Nile virus, enterovirus, and Chikungunya virus. Autoimmune testing including serum and CSF neuromyelitis optica antibodies were negative.

\section{Questions for consideration:}

1. What is the final diagnosis?

2. How would you treat this patient?

Figure 2 Brain and spine MRI
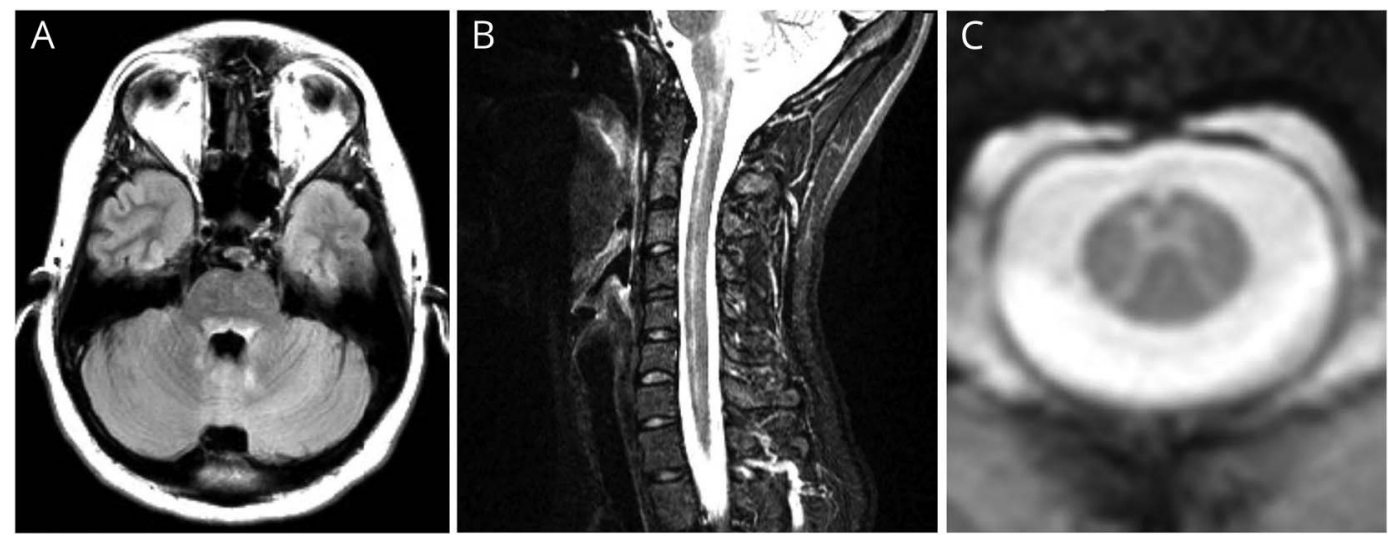

(A) Axial T2 fluid-attenuated inversion recovery brain MRI reveals T2 confluent hyperintensity in the dorsal pons and surrounding the 4th ventricle. (B) Sagittal T2 STIR cervical spine MRI shows linear T2 hyperintensity spanning the length of the anterior portion of the cervical cord. (C) T2 axial spine MRI at the level of the C2 shows T2 hyperintensity forming an outline of the anterior horn cells and gray matter structures. 


\section{Section 4}

With the positive serum $M$ pneumoniae IgM and confirmatory immunofluorescence test, we diagnosed the patient with mycoplasma encephalomyelitis.

The patient received a 5-day course of azithromycin beginning hospital day 3 (empiric treatment initially, which continued when serum serologies for mycoplasma returned positive). Given the lack of mycoplasma DNA in her CSF, the timing of her neurologic symptoms, and a positive IgM serology for $M$ pneumoniae, we suspected a primarily immune-mediated parainfectious pathophysiology. Consequently, she also received a 5-day course of IV immunoglobulin (IVIg).

The patient's symptoms rapidly improved with treatment. By the time of discharge (hospital day 9), her vertical diplopia was stable and her right arm strength improved such that she could lift the arm antigravity without difficulty.

At 3-month follow-up, the patient had no diplopia, a subtle right hyperphoria, and only subtle residual right arm weakness. She noticed difficulty fully opening the left side of her jaw-she was unable to open her mouth wide enough to eat hamburgers. Examination revealed wasting of the left masseter muscle and deviation of her jaw to the left. Review of her in-hospital MRI showed T2 hyperintensity in the area of the motor nucleus of cranial nerve $\mathrm{V}$ in the pons, which accounted for difficulty with mastication (figure 3).

\section{Discussion}

$M$ pneumoniae has been associated with a diverse range of neuropathologies that can affect any location in the neuraxis including central (encephalitis, acute disseminated encephalomyelitis, transverse myelitis, striatal necrosis, aseptic meningitis, opsoclonus-myoclonus, optic neuritis, strokes, and seizures) as well as peripheral (Guillain-Barré syndrome, polyradiculitis, and cranial nerve palsies). Encephalitis, a common manifestation, is more frequently reported in children than adults. ${ }^{4}$ CNS demyelination is a frequent pathologic endpoint in mycoplasma infections, which can cause a life-threatening acute disseminated encephalomyelitis. Demyelination may also affect the spine alone, presenting as transverse myelitis. ${ }^{4,5}$ In addition to skew deviation, ocular presentations of $M$ pneumoniae include opsoclonusmyoclonus, optic neuritis, and neuroretinitis. ${ }^{6} \mathrm{M}$ pneumoniae has a predilection for the brainstem and has been implicated in cranial nerve palsies as well as Bickerstaff brainstem encephalitis.

Several possible mechanisms may account for the wide variety of neurologic manifestations associated with $M$ pneumoniae. Some studies propose direct bacterial invasion of the CNS,, 4 while others postulate an immune-mediated, parainfectious effect, citing autoantibody production as the primary mode of injury. ${ }^{6}$ Alternatively, immune complex deposition may cause CNS vasculitis and tissue ischemia. ${ }^{5}$

Given the lack of mycoplasma DNA in our patient's CSF and the timing of her neurologic symptoms, we suspected an immune-mediated, parainfectious pathogenesis. Indeed, $M$ pneumoniae is often not detected in CSF in cases of mycoplasma encephalitis; one study estimates that DNA is only detected in CSF in up to $14 \%$ of cases. ${ }^{6}$ A neutrophilpredominant vs lymphocyte-predominant pleocytosis has also been postulated to indicate direct bacterial invasion vs parainfectious immune-mediated mechanisms. ${ }^{7}$ In our case, we observed an increasing pleocytosis between hospital day 1 and day 4 with a shift from neutrophil-predominant to lymphocyte-predominant CSF. This shift potentially favors a parainfectious mechanism, but this is currently speculative.

No randomized trials exist to guide management of the neurologic complications of $M$ pneumoniae infection. Antibiotics and immunomodulating therapy are considered the cornerstones of therapy. It is unknown if early treatment with antibiotics can prevent neurologic sequelae. However, since the bacterium itself may be responsible for CNS damage, antibiotic treatment is recommended. Immunomodulating therapies have been implemented with varying degrees of treatment success. Case reports highlight a role for
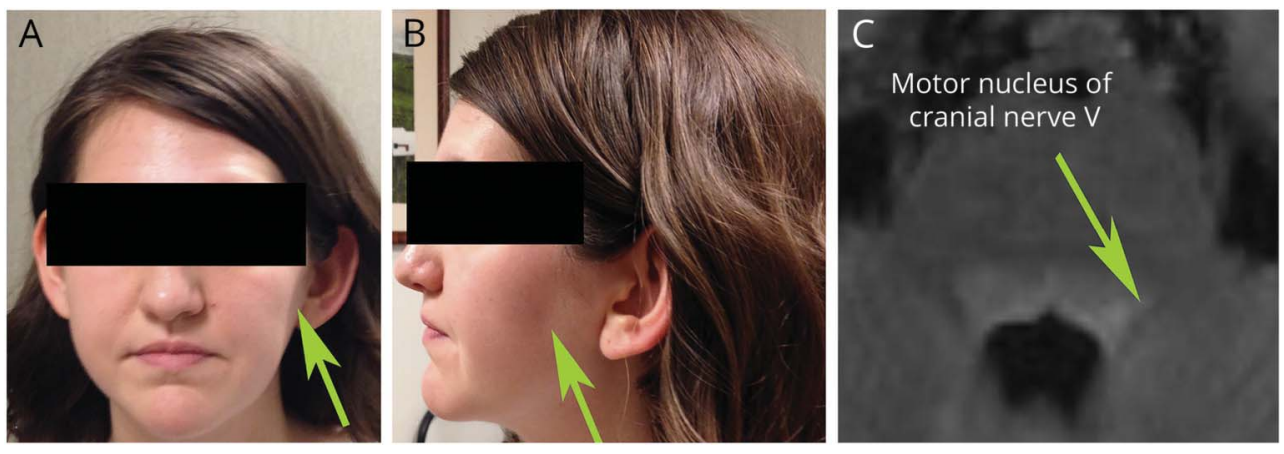

At 3-month follow-up, the patient complained of difficulty opening her jaw. Physical examination revealed wasting of the patient's left masseter muscle (A, B). Review of her in-hospital MRI revealed T2 hyperintensity in the area of the motor nucleus of cranial nerve $\mathrm{V}$ motor nucleus (C). 
corticosteroids. ${ }^{8}$ Plasma exchange has also been tried, but usually in cases of IVIg treatment failure. ${ }^{9}$

Our case highlights the utility of IVIg in modulating the suspected immune-mediated injury in patients with $M$ pneumoniae-related neurologic injury. Rationale for the use of immunoglobulin derives from nonspecific inhibition of multiple inflammatory pathways. In our case, early initiation of IVIg was associated with rapid improvement of symptoms. Prior case reports have also documented swift recovery and long-term resolution of neurologic symptoms after treatment with IVIg. ${ }^{9,10}$ This report adds to the growing body of cases supporting a role for IVIg in shortening the duration and severity of $M$ pneumoniae parainfectious neurologic complications.

\section{Author contributions}

David J. Lin: manuscript concept, drafting, revision. Seth N. Levin: manuscript drafting and revision. Catherine S.W. Albin: manuscript drafting and revision. Anna E. Goodheart: manuscript drafting and revision. Nagagopal Venna: critical revision of manuscript for intellectual content.

\section{Study funding}

No targeted funding reported.

\section{Disclosure}

D. Lin is a consultant for Boehringer Ingelheim International.

S. Levin, C. Albin, A. Goodheart, and N. Venna report no disclosures relevant to the manuscript. Go to Neurology.org/ $\mathrm{N}$ for full disclosures.

\section{References}

1. Brandt TH, Dieterich M. Different types of skew deviation. J Neurol Neurosurg Psychiatry 1991;54:549-550.

2. Brodsky MC, Donahue SP, Vaphiades M, Brandt T. Skew deviation revisited. Surv Ophthalmol 2006;51:105-128.

3. Wong AMF, Colpa L, Chandrakumar M. Ability of an upright-supine test to differentiate skew deviation from other vertical strabismus causes. Arch Ophthalmol 2011; 129:1570-1575.

4. Abramovitz P, Schvartzman P, Harel D, Lis I, Naot Y. Direct invasion of the central nervous system by Mycoplasma pneumoniae: a report of two cases. J Infect Dis 1987;155:482-487.

5. Narita M. Pathogenesis of neurologic manifestations of mycoplasma pneumoniae infection. Pediatr Neurol 2009;41:159-166.

6. Meyer Sauteur PM, Jacobs BC, Spuesens EBM, et al. Antibody responses to Mycoplasma pneumoniae: role in pathogenesis and diagnosis of encephalitis? PLoS Pathog 2014;10:e1003983.

7. Daxboeck F, Blacky A, Seidl R, Krause R, Assadian O. Diagnosis, treatment, and prognosis of Mycoplasma pneumoniae childhood encephalitis: systematic review of 58 cases. J Child Neurol 2004;19:865-871.

8. Carpenter TC. Corticosteroids in the treatment of severe mycoplasma encephalitis in children. Crit Care Med 2002;30:925-927.

9. Chambert-Loir C, Ouachee M, Collins K, Evrard P, Servais L. Immediate relief of Mycoplasma pneumoniae encephalitis symptoms after intravenous immunoglobulin. Pediatr Neurol 2009;41:375-377.

10. Gorman MP, Rincon SP, Pierce VM. Case records of the Massachusetts General Hospital: case 19-2014: a 19-year-old woman with headache, fever, stiff neck, and mental-status changes. N Engl J Med 2014;370:2427-2438. 


\section{Neurology}

\section{Clinical Reasoning: A 23-year-old woman with fever and vertical diplopia}

David J. Lin, Seth N. Levin, Catherine S.W. Albin, et al.

Neurology 2018;90;e2006-e2010

DOI 10.1212/WNL.0000000000005600

\section{This information is current as of May 28, 2018}

\section{Updated Information \& Services}

References

Subspecialty Collections

Permissions \& Licensing

Reprints including high resolution figures, can be found at: http://n.neurology.org/content/90/22/e2006.full

This article cites 10 articles, 1 of which you can access for free at: http://n.neurology.org/content/90/22/e2006.full\#ref-list-1

This article, along with others on similar topics, appears in the following collection(s):

Clinical neurology examination

http://n.neurology.org/cgi/collection/clinical_neurology_examination Diplopia (double vision)

http://n.neurology.org/cgi/collection/diplopia_double_vision

Information about reproducing this article in parts (figures,tables) or in its entirety can be found online at:

http://www.neurology.org/about/about_the_journal\#permissions

Information about ordering reprints can be found online:

http://n.neurology.org/subscribers/advertise

Neurology ${ }^{\circledR}$ is the official journal of the American Academy of Neurology. Published continuously since 1951, it is now a weekly with 48 issues per year. Copyright @ 2018 American Academy of Neurology. All rights reserved. Print ISSN: 0028-3878. Online ISSN: 1526-632X.

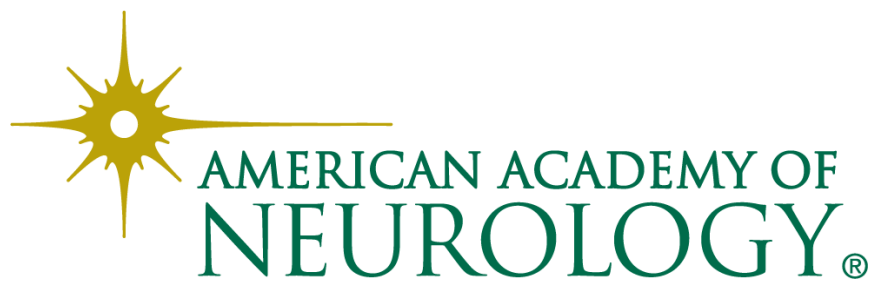

